$\beta$-Change of Riemannian Space and Special Cases of one-form $\beta$

\title{
$\beta$-Change of Riemannian Space and Special Cases of one-form $\beta$
}

\author{
Khageshwar Mandal \\ Department of Mathematics \& Statistics, Padma Kanya Multiple Campus, Kathmandu \\ Tribhuvan University, Nepal \\ Correspondence to: Khageshwar Mandal, email: khageshwarmandal@gmail.com
}

\begin{abstract}
In this paper, we consider $\beta$-change of Finsler metric $L$ given by $\bar{L}=f(L, \beta)$, where $f$ is any positively homogeneous function of degree one in $L$ and $\beta$. The objectives of the paper are to obtain the $\beta$-change of a Riemannian space, projective change of Finsler metric and to discuss the special cases of one-form $\beta$.
\end{abstract}

Keywords: $\beta$-change, Riemannian space, Finsler metric

\section{Introduction}

Let $F^{n}=\left(M^{n}, L\right)$ be an $n$-dimensional Finsler space on a differentiable manifold $M^{n}$, equipped with the fundamental function $L(x, y)$. In 2012, Shukla et al. [8] introduced the transformation of Finsler metric called Matsumoto change of metric given by

$$
\bar{L}(x, y)=\frac{L^{2}}{L-\beta}
$$

where $\beta(x, y)=b_{i}(x) y^{i}$ is a one-form on $M^{n}$. They obtained the necessary and sufficient condition for this change of Finsler metric to be projective change.

Prasad et al. [7] introduced an exponential change of Finsler metric given by

$$
\bar{L}(x, y)=L e^{\beta / L}
$$

and they dealt with the imbedding class numbers of the tangent Riemannian space of corresponding spaces.

Recently, Prasad and Kumari [6] introduced the $\beta$-change of Finsler space given by

$$
\bar{L}(x, y)=f(L, \beta),
$$

where $f$ is positively homogeneous function of $y^{i}$ of degree one in $L$ and $\beta$. They also dealt with the imbedding classes of the tangent Riemannian spaces. We have considered the same $\beta$-change given by the equation (1.1) and obtained the necessary and sufficient condition under which this change becomes a projective change. The particular cases when the vector field $b_{i}$ in $\beta$ is special one have been discussed. The Berwald's connection coefficients for the $\beta$-changed space have been calculated.

\section{Preliminaries}

Let $F^{n}=\left(M^{n}, L\right)$ be a Finsler space equipped with the fundamental function $L(x, y)$ on the smooth manifold $M^{n}$. Let $\beta=b_{i}(x) y^{i}$ be a one-form on the manifold $M^{n}$, then $L \rightarrow f(L, \beta)$ is the $\beta$-change of Finsler metric. If we write $\bar{L}=f(L, \beta)$, where $f$ is any positively homogeneous function of degree one in $L$ and $\beta$ and $\bar{F}^{n}=\left(M^{n}, \bar{L}\right)$, then the Finsler space $\bar{F}^{n}$ is said to be obtained from $F^{n}$ by $\beta$-change. The quantities corresponding to $\bar{F}^{n}$ are denoted by putting bar on those quantities.

The fundamental metric tensor $g_{i j}$, the normalized element of support $l_{i}$ and angular metric tensor $h_{i j}$ of $F^{n}$ are given by

$$
g_{i j}=\frac{1}{2} \frac{\partial^{2} L^{2}}{\partial y^{i} \partial y^{j}}, \quad l_{i}=\frac{\partial L}{\partial y^{i}} \quad \text { and } \quad h_{i j}=L \frac{\partial^{2} L}{\partial y^{i} \partial y^{j}}=g_{i j}-l_{i} l_{j}
$$


We shall denote the partial derivative with respect to $x^{i}$ and $y^{i}$ by $\partial_{i}$ and $\dot{\partial}_{i}$ respectively and write

$$
L_{i}=\dot{\partial}_{i} L, \quad L_{i j}=\dot{\partial}_{i} \dot{\partial}_{j} L, \quad L_{i j k}=\dot{\partial}_{i} \dot{\partial}_{j} \dot{\partial}_{k} L
$$

Then,

$$
L_{i}=l_{i}, \quad L^{-1} h_{i j}=L_{i j}
$$

The geodesic of $F^{n}$ are given by the system of differential equations

$$
\frac{d^{2} x^{i}}{d s^{2}}+2 G^{i}\left(x, \frac{d x}{d s}\right)=0
$$

where $G^{i}(x, y)$ are positively homogeneous of degree two in $y^{i}$ and are given by

$$
2 G^{i}=g^{i j}\left(y^{r} \dot{\partial}_{j} \partial_{r} F-\partial_{j} F\right), \quad F=\frac{L^{2}}{2}
$$

where $g^{i j}$ are the inverse of $g_{i j}$. The well known Berwald connection $B \Gamma=\left(G_{j k}^{i}, G_{j}^{i}\right)$ of a Finsler space is constructed from the quantity $G^{i}$ appearing in the equation of geodesic and is given by 8 ]

$$
G_{j}^{i}=\dot{\partial}_{j} G^{i}, \quad G_{j k}^{i}=\dot{\partial}_{k} G_{j}^{i}
$$

The Cartan's connection $C \Gamma=\left(F_{j k}^{i}, G_{j}^{i}, C_{j k}^{i}\right)$ is constructed from the metric function $L$ by the following five axioms [8]:
(i) $g_{i j \mid k}=0$
(ii) $\left.\quad g_{i j}\right|_{k}=0$
(iii) $\quad F_{j k}^{i}=F_{k j}^{i}$
(iv) $F_{0 k}^{i}=G_{k}^{i}$
(v) $C_{j k}^{i}=C_{k j}^{i}$,

where $\left.\right|_{k}$ and $\left.\right|_{k}$ denote $h$ - and $v$-covariant derivatives with respect to $C \Gamma$. It is clear that the $h$-covariant derivative of $L$ with respect to $B \Gamma$ and $C \Gamma$ is the same and vanishes identically. Furthermore, the $h$-covariant derivatives of $L_{i}, L_{i j}$ with respect to $C \Gamma$ are also zero.

We shall write

$$
2 r_{i j}=b_{i \mid j}+b_{j \mid i}, \quad 2 s_{i j}=b_{i \mid j}-b_{j \mid i}
$$

\section{The $\beta$-change of Finsler Metric}

The $\beta$-change of Finsler metric is given by

$$
\bar{L}(x, y)=f(L, \beta),
$$

where $f$ is positively homogeneous function of degree one in $L$ and $\beta$. Homogeneity of $f$ gives

$$
L f_{1}+\beta f_{2}=f,
$$

where subscripts ' 1 ' and ' 2 ' denote the partial derivatives with respect to $L$ and $\beta$ respectively.

Differentiating 3.2 with respect to $L$ and $\beta$ respectively, we get

$$
L f_{11}+\beta f_{12}=0 \quad \text { and } \quad L f_{12}+\beta f_{22}=0 .
$$

Hence, we have

$$
\frac{f_{11}}{\beta^{2}}=-\frac{f_{12}}{\beta L}=\frac{f_{22}}{L^{2}},
$$

which gives

$$
f_{11}=\beta^{2} \omega, \quad f_{22}=L^{2} \omega, \quad f_{12}=-\beta L \omega,
$$


where Weierstrass function $\omega$ is positively homogeneous function of degree -3 in $L$ and $\beta$. Therefore,

$$
L \omega_{1}+\beta \omega_{2}+3 \omega=0 .
$$

Again, $\omega_{2}$ is positively homogeneous of degree -4 in $L$ and $\beta$, so

$$
L \omega_{21}+\beta \omega_{22}+4 \omega_{2}=0
$$

Throughout the paper we frequently use the equations $(3.2),(3.3)$ and $(3.4)$ without quoting them. Also, we have assumed that $f$ is not linear function of $L$ and $\beta$ so that $\omega \neq 0$.

We may put

$$
\bar{G}^{i}=G^{i}+D^{i}
$$

Then, $\bar{G}_{j}^{i}=G_{j}^{i}+D_{j}^{i}$ and $\bar{G}_{j k}^{i}=G_{j k}^{i}+D_{j k}^{i}$, where $D_{j}^{i}=\dot{\partial}_{j} D^{i}$ and $D_{j k}^{i}=\dot{\partial}_{k} D_{j}^{i}$. The tensors $D^{i}, D_{j}^{i}$ and $D_{j k}^{i}$ are positively homogeneous in $y^{i}$ of degree two, one and zero respectively. Therefore, we have

$$
D_{j k}^{i} y^{k}=D_{j}^{i}, \quad D_{j}^{i} y^{j}=2 D^{i} .
$$

To find difference tensor $D^{i}$, we deal with equation [8] $L_{i j \mid k}=0$, that means,

$$
\partial_{k} L_{i j}-L_{i j r} G_{k}^{r}-L_{r j} F_{i k}^{r}-L_{i r} F_{j k}^{r}=0 .
$$

Since $\dot{\partial}_{i} \beta=b_{i}$, from 3.1 , we have

$$
\begin{aligned}
(a) \quad \bar{L}_{i}= & f_{1} L_{i}+f_{2} b_{i}, \\
(b) \quad \bar{L}_{i j}= & f_{1} L_{i j}+\beta^{2} \omega L_{i} L_{j}-\beta L \omega\left(L_{i} b_{j}+L_{j} b_{i}\right)+L^{2} \omega b_{i} b_{j}, \\
(c) \quad \partial_{j} \bar{L}_{i}= & f_{1} \partial_{j} L_{i}+\left(\beta^{2} \omega L_{i}-\beta L \omega b_{i}\right) \partial_{j} L+\left(L^{2} \omega b_{i}-\beta L \omega L_{i}\right) \partial_{j} \beta+f_{2} \partial_{j} b_{i}, \\
(d) \quad \partial_{k} \bar{L}_{i j}= & f_{1} \partial_{k} L_{i j}+\left\{\beta^{2} \omega L_{i j}+\beta^{2} \omega_{1} L_{i} L_{j}-\left(\beta L \omega_{1}+\beta \omega\right)\left(L_{i} b_{j}+L_{j} b_{i}\right)+\right. \\
& \left.\left(2 L \omega+L^{2} \omega_{1}\right) b_{i} b_{j}\right\} \partial_{k} L+\left\{-\beta L \omega L_{i j}+\left(2 \beta \omega+\beta^{2} \omega_{2}\right) L_{i} L_{j}-\right. \\
& \left.\left(L \omega_{1}+\beta L \omega_{2}\right)\left(L_{i} b_{j}+L_{j} b_{i}\right)+L^{2} \omega_{2} b_{i} b_{j}\right\} \partial_{k} \beta+ \\
& \left(\beta^{2} \omega L_{j}-\beta L \omega b_{j}\right) \partial_{k} L_{i}+\left(\beta^{2} \omega L_{i}-\beta L \omega b_{i}\right) \partial_{k} L_{j}- \\
& \left(\beta L \omega L_{j}-L^{2} \omega b_{j}\right) \partial_{k} b_{i}-\left(\beta L \omega L_{i}-L^{2} \omega b_{i}\right) \partial_{k} b_{j}, \\
(e) \quad & f_{1} L_{i j k}+\beta^{2} \omega\left(L_{i} L_{j k}+L_{j} L_{i k}+L_{k} L_{i j}\right)-\beta L \omega\left(b_{i} L_{j k}+b_{j} L_{i k}+\right. \\
& \left.b_{k} L_{i j}\right)+\left(\beta^{2} \omega_{2}+2 \beta \omega\right)\left(L_{i} L_{j} b_{k}+L_{i} L_{k} b_{j}+L_{j} L_{k} b_{i}\right)- \\
& \left(\beta L \omega_{2}+L \omega\right)\left(b_{i} b_{j} L_{k}+b_{j} b_{k} L_{i}+b_{i} b_{k} L_{j}\right)+\beta^{2} \omega_{1} L_{i} L_{j} L_{k}+L^{2} \omega_{2} b_{i} b_{j} b_{k} .
\end{aligned}
$$

Since $\bar{L}_{i j \mid k}=0$ in $\bar{F}^{n}$, after using 3.5 , we have

$$
\partial_{k} \bar{L}_{i j}-\bar{L}_{i j r}\left(G_{k}^{r}+D_{k}^{r}\right)-\bar{L}_{r j}\left(F_{i k}^{r}+{ }^{c} D_{i k}^{r}\right)-\bar{L}_{i r}\left(F_{j k}^{r}+{ }^{c} D_{j k}^{r}\right)=0,
$$

where $\bar{F}_{j k}^{i}-F_{j k}^{i}={ }^{c} D_{j k}^{i}$.

Substituting in the equation (3.8) the values of $\partial_{k} \bar{L}_{i j}, \bar{L}_{i r}$ and $\bar{L}_{i j r}$ from (3.7)(b),(d),(e) and using (3.6), 
we have

$$
\begin{aligned}
&- f_{1}\left\{L_{i j r} D_{k}^{r}+L_{r j} D_{i k}^{r}+L_{i r} D_{j k}^{r}\right\}+\left\{\beta^{2} \omega L_{i j}+\beta^{2} \omega_{1} L_{i} L_{j}-\right. \\
&\left.\left(\beta L \omega_{1}+\beta \omega\right)\left(L_{i} b_{j}+L_{j} b_{i}\right)+\left(2 L \omega+L^{2} \omega_{1}\right) b_{i} b_{j}\right\} L_{r} G_{k}^{r}+ \\
&\left\{-\beta L \omega L_{i j}+\left(2 \beta \omega+\beta^{2} \omega_{2}\right) L_{i} L_{j}-\left(L \omega+\beta L \omega_{2}\right) \times\right. \\
&\left.\left(L_{i} b_{j}+L_{j} b_{i}\right)+L^{2} \omega_{2} b_{i} b_{j}\right\}\left(r_{0 k}+s_{0 k}+b_{r} G_{k}^{r}\right)+\left(\beta^{2} \omega L_{j}-L \beta \omega b_{j}\right) \times \\
&\left(L_{i r} G_{k}^{r}+L_{r} F_{i k}^{r}\right)+\left(\beta^{2} \omega L_{i}-L \beta \omega b_{i}\right)\left(L_{j r} G_{k}^{r}+L_{r} F_{j k}^{r}\right)- \\
&\left(L \beta \omega L_{j}-L^{2} \omega b_{j}\right)\left(r_{i k}+s_{i k}+b_{r} F_{i k}^{r}\right)- \\
&\left(L \beta \omega L_{i}-L^{2} \omega b_{i}\right)\left(r_{j k}+s_{j k}+b_{r} F_{j k}^{r}\right)- \\
&\left\{\beta^{2} \omega\left(L_{i} L_{j r}+L_{j} L_{r i}+L_{r} L_{i j}\right)-L \beta \omega\left(b_{i} L_{j r}+b_{j} L_{i r}+b_{r} L_{i j}\right)+\right. \\
&\left(\beta^{2} \omega_{2}+2 \beta \omega\right)\left(L_{i} L_{j} b_{r}+L_{i} L_{r} b_{j}+L_{j} L_{r} b_{i}\right)- \\
&\left(L \beta \omega_{2}+L \omega\right)\left(L_{r} b_{i} b_{j}+L_{i} b_{j} b_{r}+L_{j} b_{i} b_{r}\right)+ \\
&\left.\beta^{2} \omega_{1} L_{i} L_{j} L_{r}+L^{2} \omega_{2} b_{i} b_{j} b_{r}\right\}\left(G_{k}^{r}+D_{k}^{r}\right)- \\
&\left\{\beta^{2} \omega L_{r} L_{j}-L \beta \omega\left(L_{r} b_{j}+L_{j} b_{r}\right)+L^{2} \omega b_{r} b_{j}\right\}\left(F_{i k}^{r}+{ }^{c} D_{i k}^{r}\right)- \\
&\left\{\beta^{2} \omega L_{r} L_{i}-L \beta \omega\left(L_{r} b_{i}+L_{i} b_{r}\right)+L^{2} \omega b_{r} b_{i}\right\}\left(F_{j k}^{r}+{ }^{c} D_{i k}^{r}\right)=0
\end{aligned}
$$

where $\partial_{k} L=L_{r} G_{k}^{r}, \partial_{k} \beta=r_{0 k}+s_{0 k}+b_{r} G_{k}^{r}, \partial_{k} L_{i}=L_{i r} G_{k}^{r}+L_{r} F_{i k}^{r}$ and $\partial_{k} b_{i}=r_{i k}+s_{i k}+b_{r} F_{i k}^{r}$.

Contracting 3.9 with $y^{k}$, and using the fact that $D_{j k}^{i} y^{j}={ }^{c} D_{j k}^{i} y^{j}=D_{k}^{i}$ [2], we get

$$
\begin{aligned}
& 2\left\{f_{1} L_{i j r}+\beta^{2} \omega\left(L_{i} L_{j r}+L_{j} L_{r i}+L_{r} L_{i j}\right\}-\right. \\
& L \beta \omega\left(b_{i} L_{j r}+b_{j} L_{i r}+b_{r} L_{i j}\right)+\left(\beta^{2} \omega_{2}+2 \beta \omega\right)\left(L_{i} L_{j} b_{r}+\right. \\
& \left.L_{i} L_{r} b_{j}+L_{j} L_{r} b_{i}\right)-\left(L \beta \omega_{2}+L \omega\right)\left(L_{r} b_{i} b_{j}+L_{i} b_{j} b_{r}+L_{j} b_{i} b_{r}\right)+ \\
& \left.\beta^{2} \omega_{1} L_{i} L_{j} L_{r}+L^{2} \omega_{2} b_{i} b_{j} b_{r}\right\} D^{r}+\left\{f_{1} L_{r j}+\beta^{2} \omega L_{r} L_{j}-\right. \\
& \left.L \beta \omega\left(L_{r} b_{j}+L_{j} b_{r}\right)+L^{2} \omega b_{r} b_{j}\right\} D_{i}^{r}+\left\{f_{1} L_{i r}+\beta^{2} \omega L_{r} L_{i}-\right. \\
& \left.L \beta \omega\left(L_{r} b_{i}+L_{i} b_{r}\right)+L^{2} \omega b_{r} b_{i}\right\} D_{j}^{r}+\left(L \beta \omega L_{j}-L^{2} \omega b_{j}\right) \times \\
& \left(r_{i 0}+s_{i 0}\right)+\left(L \beta \omega L_{i}-L^{2} \omega b_{i}\right)\left(r_{j 0}+s_{j 0}\right)+\left\{\beta L \omega L_{i j}-\left(2 \beta \omega+\beta^{2} \omega_{2}\right) L_{i} L_{j}+\right. \\
& \left.\left(L \omega+L \beta \omega_{2}\right)\left(L_{i} b_{j}+L_{j} b_{i}\right)-L^{2} \omega_{2} b_{i} b_{j}\right\} r_{00}=0,
\end{aligned}
$$

where ${ }^{\prime} 0^{\prime}$ stands for contraction with respect to $y^{i}$, viz. $r_{0 k}=r_{i k} y^{i}, r_{00}=r_{i j} y^{i} y^{j}$.

Next, we deal with $\bar{L}_{i \mid j}=0$, that is $\partial_{j} \bar{L}_{i}-\bar{L}_{i r} \bar{G}_{j}^{r}-\bar{L}_{r} \bar{F}_{i j}^{r}=0$, then

$$
\partial_{j} \bar{L}_{i}-\bar{L}_{i r}\left(G_{j}^{r}+D_{j}^{r}\right)-\bar{L}_{r}\left(F_{i j}^{r}+{ }^{c} D_{i j}^{r}\right)=0 .
$$

Putting the values of $\partial_{j} \bar{L}_{i}, \bar{L}_{i r}$ and $\bar{L}_{r}$ from 3.7 in 3.11 and using equation

$$
L_{i \mid j}=\partial_{j} L_{i}-L_{i r} G_{j}^{r}-L_{r} F_{i j}^{r}=0
$$

and rearranging the terms, we get

$$
\begin{aligned}
f_{2} b_{i \mid j}=\{ & \left.f L_{i r}+\beta^{2} \omega L_{i} L_{r}+L^{2} \omega b_{i} b_{r}-L \beta \omega\left(L_{i} b_{r}+L_{r} b_{i}\right)\right\} D_{j}^{r} \\
& +\left(L \beta \omega L_{i}-L^{2} \omega b_{i}\right)\left(r_{0 j}+s_{0 j}\right)+\left(f_{1} L_{r}+f_{2} b_{r}\right) D_{i j}^{r},
\end{aligned}
$$

which after using 2.2 gives

$$
\begin{aligned}
2 f_{2} r_{i j}= & \left\{f_{1} L_{i r}+\beta^{2} \omega L_{i} L_{r}+L^{2} \omega b_{i} b_{r}-L \beta \omega\left(L_{i} b_{r}+L_{r} b_{i}\right)\right\} D_{j}^{r}+ \\
& \left\{f_{1} L_{j r}+\beta^{2} \omega L_{j} L_{r}+L^{2} \omega b_{j} b_{r}-L \beta \omega\left(L_{j} b_{r}+L_{r} b_{j}\right)\right\} D_{i}^{r}+ \\
& \left(L \beta \omega L_{i}-L^{2} \omega b_{i}\right)\left(r_{0 j}+s_{0 j}\right)+\left(L \beta \omega L_{j}-L^{2} \omega b_{j}\right) \times \\
& \left(r_{0 i}+s_{0 i}\right)+2\left(f_{1} L_{r}+f_{2} b_{r}\right) D_{i j}^{r}
\end{aligned}
$$


and

$$
\begin{aligned}
2 f_{2} s_{i j}= & \left\{f_{1} L_{i r}+\beta^{2} \omega L_{i} L_{r}+L^{2} \omega b_{i} b_{r}-L \beta \omega\left(L_{i} b_{r}+L_{r} b_{i}\right)\right\} D_{j}^{r}- \\
& \left\{f_{1} L_{j r}+\beta^{2} \omega L_{j} L_{r}+L^{2} \omega b_{j} b_{r}-L \beta \omega\left(L_{j} b_{r}+L_{r} b_{j}\right)\right\} D_{i}^{r}+ \\
& \left(L \beta \omega L_{i}-L^{2} \omega b_{i}\right)\left(r_{0 j}+s_{0 j}\right)-\left(L \beta \omega L_{j}-L^{2} \omega b_{j}\right)\left(r_{0 i}+s_{0 i}\right) .
\end{aligned}
$$

Subtracting 3.12 from 3.10 and contracting the resulting equation with $y^{i}$, we obtain

$$
\begin{aligned}
& \left\{-f_{1} L_{j r}+L \beta \omega L_{j} b_{r}+L \beta \omega L_{r} b_{j}-\beta^{2} \omega L_{j} L_{r}-L^{2} \omega b_{j} b_{r}\right\} D^{r}- \\
& \frac{1}{2}\left(L \beta \omega L_{j}-L^{2} \omega b_{j}\right) r_{00}+f_{2} r_{0 j}=\left(f_{1} L_{r}+f_{2} b_{r}\right) D_{j}^{r} .
\end{aligned}
$$

Contracting 3.14 with $y^{j}$, we get

$$
2\left(f_{1} L_{r}+f_{2} b_{r}\right) D^{r}=f_{2} r_{00} .
$$

Adding 3.10 and 3.13 and contracting the resulting equation with $y^{j}$, we get

$$
\left\{f_{1} L_{i r}+\beta^{2} \omega L_{i} L_{r}+L^{2} \omega b_{i} b_{r}-L \beta \omega\left(L_{i} b_{r}+L_{r} b_{i}\right)\right\} D^{r}=\frac{1}{2}\left(L^{2} \omega b_{i}-L \beta \omega L_{i}\right) r_{00}+f_{2} s_{i 0}
$$

In view of $L L_{i r}=g_{i r}-L_{i} L_{r}$, the equation 3.16 can be written as

$$
\begin{aligned}
& \frac{f_{1}}{L} g_{i r} D^{r}+\left\{\left(-\frac{f_{1}}{L}+\beta^{2} \omega\right) L_{i}-L \beta \omega b_{i}\right\} L_{r} D^{r}+ \\
& \left(L^{2} \omega b_{i}-L \beta \omega L_{i}\right) b_{r} D^{r}=\frac{1}{2}\left(L^{2} \omega b_{i}-L \beta \omega L_{i}\right) r_{00}+f_{2} s_{i 0}
\end{aligned}
$$

Contracting (3.17) with $b^{i}=g^{i j} b_{j}$, we get

$$
\left(\frac{-f_{1} \beta}{L^{2}}-L \beta \omega \triangle\right) L_{r} D^{r}+\left(\frac{f_{1}}{L}+L^{2} \omega \triangle\right) b_{r} D^{r}=\frac{L^{2} \omega \triangle}{2} r_{00}+f_{2} s_{0},
$$

where $\triangle=b^{2}-\frac{\beta^{2}}{L^{2}}$ and $s_{0}=s_{r 0} b^{r}$.

The equations $(3.15)$ ) and 3.18 constitute the system of algebraic equations in $L_{r} D^{r}$ and $b_{r} D^{r}$ whose solution is given by

$$
b_{r} D^{r}=\frac{\left(f_{1} f_{2} \beta+f L^{3} \omega \triangle\right)}{2 f\left(f_{1}+L^{3} \omega \triangle\right)} r_{00}+\frac{f_{1} f_{2} L^{2}}{f\left(f_{1}+L^{3} \omega \triangle\right.} s_{0}
$$

and

$$
L_{r} D^{r}=\frac{L f_{1} f_{2}}{2 f\left(f_{1}+L^{3} \omega \triangle\right)} r_{00}-\frac{L^{2} f_{2}^{2}}{f\left(f_{1}+L^{3} \omega \triangle\right)} s_{0} .
$$

Contracting (3.17) by $g^{i j}$ and putting the values of $b_{r} D^{r}$ and $L_{r} D^{r}$ from 3.19 and 3.20 respectively, we get

$$
\begin{aligned}
D^{i}= & \left\{\frac{f_{1} f_{2}-f L \beta \omega}{2 f\left(f_{1}+L^{3} \omega \triangle\right)} r_{00}-\frac{L f_{2}\left(f_{1} f_{2}-f L \beta \omega\right)}{f f_{1}\left(f_{1}+L^{3} \omega \triangle\right)} s_{0}\right\} y^{i}+ \\
& \left\{\frac{L^{3} \omega}{2\left(f_{1}+L^{3} \omega \triangle\right)} r_{00}-\frac{f_{2} L^{4} \omega}{f_{1}\left(f_{1}+L^{3} \omega \triangle\right)} s_{0}\right\} b^{i}+\frac{L f_{2}}{f_{1}} s_{0}^{i}
\end{aligned}
$$

where $l^{i}=\frac{y^{i}}{L}$.

Proposition 3.1. The difference tensor $D^{i}=\bar{G}^{i}-G^{i}$ of any $\beta$-change of Finsler metric is given by (3.21. 


\section{Projective Change of Finsler Metric}

The Finsler space $\bar{F}^{n}$ is said to be projective to Finsler space $F^{n}$ if every geodesic of $F^{n}$ is transformed to a geodesic of $\bar{F}^{n}$. It is well known that the change $L \rightarrow \bar{L}$ is projective if $G^{i}=G^{i}+P(x, y) y^{i}$, where $P(x, y)$ is a homogeneous scalar function of degree one in $y^{i}$, called projective factor [3]. Thus, from (3.5) it follows that $L \rightarrow \bar{L}$ is projective iff $D^{i}=P y^{i}$.

Now, we consider that the $\beta$-change $L \rightarrow \bar{L}=f(L, \beta)$ is projective. Then, from equation 3.21, we have

$$
\begin{aligned}
P y^{i}= & \left\{\frac{f_{1} f_{2}-f L \beta \omega}{2 f\left(f_{1}+L^{3} \omega \triangle\right)} r_{00}-\frac{L f_{2}\left(f_{1} f_{2}-f L \beta \omega\right)}{f f_{1}\left(f_{1}+L^{3} \omega \triangle\right)} s_{0}\right\} y^{i}+ \\
& \left\{\frac{L^{3} \omega}{2\left(f_{1}+L^{3} \omega \triangle\right)} r_{00}-\frac{f_{2} L^{4} \omega}{f_{1}\left(f_{1}+L^{3} \omega \triangle\right)} s_{0}\right\} b^{i}+\frac{L f_{2}}{f_{1}} s_{0}^{i},
\end{aligned}
$$

Contracting (4.1) with $y_{i}\left(=g_{i j} y^{j}\right)$ and using the fact that $s_{0}^{i} y_{i}=0$ and $y_{i} y^{i}=L^{2}$, we get

$$
P=\frac{f_{1} f_{2}}{2 f\left(f_{1}+L^{3} \omega \triangle\right)} r_{00}-\frac{f_{2}^{2} L}{f\left(f_{1}+L^{3} \omega \triangle\right)} s_{0} .
$$

Putting the value of $P$ from 4.2 in 4.1 , we get

$$
\beta \omega\left(f_{1} r_{00}-2 f_{2} L s_{0}\right) y^{i}=L^{2} \omega\left(f_{1} r_{00}-2 f_{2} L s_{0}\right) b^{i}+2 f_{2}\left(f_{1}+L^{3} \omega \triangle\right) s_{0}^{i} .
$$

Transvecting 4.3 by $b_{i}$, we get

$$
r_{00}=\frac{-2 f_{2}}{L^{2} \omega} \frac{s_{0}}{\triangle}, \quad \text { where } \quad \triangle=b^{2}-\frac{\beta^{2}}{L^{2}} \neq 0
$$

Substituting the value of $r_{00}$ from 4.4 in 4.2, we get

$$
P=\frac{-f_{2}^{2}}{f L^{2} \omega} \frac{s_{0}}{\triangle}
$$

Eliminating $P$ and $r_{00}$ from (4.5), 4.4) and 4.1), we get

$$
s_{0}^{i}=\left(b^{i}-\frac{\beta}{L^{2}} y^{i}\right) \frac{s_{0}}{\triangle} .
$$

The equations 4.4 and 4.6 give the necessary conditions under which the $\beta$-change becomes a projective change. Conversely, if conditions (4.4) and (4.6) are satisfied, then putting these conditions in (3.21), we get

$$
D^{i}=\frac{-f_{2}^{2}}{f L^{3} \omega} \frac{s_{0}}{\triangle} y^{i}, \quad \text { i.e. } \quad D^{i}=P y^{i}, \quad \text { where } P=\frac{-f_{2}^{2}}{f L^{3} \omega} \frac{s_{0}}{\triangle} .
$$

Thus $\bar{F}^{n}$ is projective to $F^{n}$.

Theorem 4.1. The $\beta$-change of Finsler space is projective if and only if (4.4) and (4.6) hold.

Let us assume that $L$ is the metric of a Riemannian space, that is, $L=\alpha=\sqrt{a_{i j}(x) y^{i} y^{j}}$. Then $\bar{L}=f(\alpha, \beta)$ which is the metric of any $\beta$-changed space. In this case $b_{i \mid j}=b_{i ; j}$ where $; j$ denotes the covariant derivative with respect to Christoffel symbols constructed from Riemannian metric $\alpha$. Thus $r_{i j}$ and $s_{i j}$ are functions of coordinates only, and in view of the Theorem 4.1, it follows that the Riemannian space is projective to Finsler space obtained from $\beta$-change 3.1 iff

$$
r_{00}=\frac{-2 f_{2}}{\alpha^{2} \omega} \frac{s_{0}}{\triangle} \quad \text { and } \quad s_{0}^{i}=-\left(\frac{\beta}{\alpha^{2}} y^{i}-b^{i}\right) \frac{s_{0}}{\triangle}, \text { where } \triangle=b^{2}-\frac{\beta^{2}}{\alpha^{2}} \neq 0
$$


These equations may be written as

$$
\text { (a) } r_{00}\left(\beta^{2}-b^{2} \alpha^{2}\right)=\frac{2 f_{2}}{\omega} s_{0}, \quad \text { (b) } s_{0}^{i}\left(\beta^{2}-b^{2} \alpha^{2}\right)=\left(\beta y^{i}-\alpha^{2} b^{i}\right) s_{0} \text {. }
$$

The equation 4.7 (b) can be written as

$$
\begin{aligned}
\left(s_{j}^{i} b_{h} b_{k}+s_{h}^{i} b_{j} b_{k}+s_{k}^{i} b_{j} b_{h}\right)-b^{2}\left(s_{j}^{i} a_{h k}+s_{h}^{i} a_{j k}+s_{k}^{i} a_{j h}\right) \\
=\frac{1}{2}\left[\left(b_{h} s_{k}+b_{k} s_{h}\right) \delta_{j}^{i}+\left(b_{j} s_{k}+b_{k} s_{j}\right) \delta_{h}^{i}+\right. \\
\left.\quad\left(b_{j} s_{h}+b_{h} s_{j}\right) \delta_{k}^{i}\right]-b^{i}\left(a_{h k} s_{j}+a_{h j} s_{k}+a_{j k} s_{h}\right) .
\end{aligned}
$$

Contracting this equation with $i=j$, we get

$$
\left(s_{h} b_{k}+s_{k} b_{h}\right)=0, \text { for } n>2 .
$$

Transvection of 4.8 by $b^{h}$, we get $b^{2} s_{k}=0$, which implies that $s_{k}=0$ provided $b^{2} \neq 0$. Therefore, we have $s_{0}^{i}=0, s_{0}=0$ and 4.7 (a) gives $r_{00}=0$ as $\beta^{2}-b^{2} \alpha^{2} \neq 0$, consequently $r_{i j}=0, s_{i j}=0$. Hence $b_{i ; j}=0$, i.e. the pair $(\alpha, \beta)$ is parallel pair. Conversely, if $b_{i ; j}=0$ the equation 4.7 (a) and (b) hold identically. Thus, we have the following theorem

Theorem 4.2. The Riemannian space with metric $\alpha$ is projective to the Finsler space with $(\alpha, \beta)-$ metric iff the $(\alpha, \beta)$ is a parallel pair.

\section{Particular Cases of One-form $\beta$}

Let $b_{i}$ be components of a parallel vector field in $F^{n}$ i.e. $b_{i \mid j}=0$. Therefore $r_{i j}=s_{i j}=0$. Hence, the equation 3.21 gives $D^{i}=0$ which implies that $\bar{G}^{i}=G^{i}, \bar{G}_{j}^{i}=G_{j}^{i}$ and $\bar{G}_{j k}^{i}=G_{j k}^{i}$. Thus, we have the following theorem

Theorem 5.1. The Berwald connection $B \Gamma=\left(G_{j k}^{i}, G_{j}^{i}, 0\right)$ is invariant under $\beta$-change for parallel vector field $b_{i}$.

Let $b_{i}$ be a concurrent vector field in $F^{n}$ [4, 9]. Then, we have (i) $b_{i \mid j}=-g_{i j}$

(ii) $b_{i} C_{j k}^{i}=0$.

Thus, for a concurrent vector field $s_{i j}=0, r_{i j}=-g_{i j}$ and therefore $r_{00}=-L^{2}$ and equation 3.21 reduces to

$$
D^{i}=-\frac{\left(f_{1} f_{2}-f L \beta \omega\right) L^{2}}{2 f\left(f_{1}+L^{3} \omega \triangle\right)} y^{i}-\frac{L^{5} \omega}{2\left(f_{1}+L^{3} \omega \triangle\right)} b^{i} .
$$

If $D^{i}=0$, then equation (5.1) shows that $b^{i}$ and $y^{i}$ are linearly related. That is, there exists a scalar $\lambda$ such that

$$
b^{i}=\lambda y^{i} .
$$

Since for a concurrent vector field the contravariant components $b^{i}$ are also functions of $x^{i}$ only [4, 9], differentiating 5.2 with respect to $y^{j}$, we get

$$
\left(\dot{\partial}_{j} \lambda\right) y^{i}+\lambda \delta_{j}^{i}=0
$$

Contracting it with respect to $i$ and $j$ and using the fact that $\lambda$ is homogeneous function of degree -1 in $y^{i}$, we get

$$
(n-1) \lambda=0 \quad \text { i.e. } \quad \lambda=0
$$

which is not possible as in that case $\beta$ vanishes. Hence, we have the following theorem 
Theorem 5.2. If $b_{i}$ are components of a concurrent vector field, then the Berwald connection B $\Gamma$ is not invariant under $\beta$-change.

Next, suppose that $b_{i}$ is a gradient vector field so that $s_{i j}=\frac{1}{2}\left(\frac{\partial b_{i}}{\partial x^{j}}-\frac{\partial b_{j}}{\partial x^{i}}\right)=0$. Then equation 3.21 reduces to

$$
D^{i}=\frac{\left(f_{1} f_{2}-f L \beta \omega\right)}{2 f\left(f_{1}+L^{3} \omega \triangle\right)} r_{00} y^{i}+\frac{L^{3} \omega r_{00}}{2\left(f_{1}+L^{3} \omega \triangle\right)} b^{i}
$$

If $r_{00} \neq 0$ and $D^{i}=0$, then we get the same result as given in theorem 5.2 for gradient vector field $b_{i}$ but if $r_{00}$ is also zero, then the Berwald connections $B \Gamma$ is invariant under $\beta$-change. Thus, we have the following theorem

Theorem 5.3. If $b_{i}$ are components of a gradient vector field, then the Berwald connection B $\Gamma$ is invariant if $r_{00}=0$.

\section{Conclusion}

Here, I introduced $n$-dimension Finsler space differentiable on a manifold along with a fundamental function. With some preliminaries and historical developments, we mainly focused on the $\beta$-change of Finsher metric and projective change of Finsler metric. The difference tensor of any $\beta$-change of Finsher metric is derived. The necessary and sufficient condition of the $\beta$-change of a Finsher space to be projective is presented. Some particular cases of $\beta$-change and Berwald connections are discussed.

Acknowledgments: This research was funded by University Grants Commission (PhD fellow 2068 BS2069 BS awarded to the author), Nepal for the financial support provided as a PhD fellowship.

\section{References}

[1] Matsumoto M (1974) On Finsler space with Randers metric and special forms of important tensors, J. Math. Kyoto Univ., 14, 477-498.

[2] Matsumoto M (1986) Foundations of Finsler geometry and special Finsler spaces, Kaiseisha Press, Otsu, Japan.

[3] Matsumoto, M (1992) Theory of Finsler spaces with $(\alpha, \beta)$ - metric, Rep. Math. Phy., 31, 43-83.

[4] Matsumoto M and Eguchi K (1974) Finsler space admitting a concurrent vector field, Tensor, N. S., 28, 239-249.

[5] Park HS and Lee IY (2001) The Randers changes of Finsler spaces with $(\alpha, \beta)-$ metrics of Douglas type, J. Korean Math. Soc., 38, 503-521.

[6] Prasad, B. N. and Kumari, B., 2013, The $\beta$-change of Finsler metric and imbedding classes of their tangent spaces, Tensor, N. S., 74(1): 48-59.

[7] Prasad BN, Shukla HS and Pandey OP (2013) The exponential change of Finsler metric and relation between imbedding class numbers of their tangent Riemannian spaces, Romanian Journal of Mathematics and Computer Science, 3(1):96-108.

[8] Shukla HS, Pandey OP and Joshi HD (2012) Matsumoto change of Finsler metric, J. Int. Acad. Phys. Sci., 16(4):329-341.

[9] Tachibana S (1950) On Finsler spaces which admit a concurrent vector field, Tensor, N. S., 1:1-5. 